\title{
ROUNDUP360
}

Wrist \& Hand

To splint or not to splint?

- Following on from December's Roundup, a further study examining the role of splinting in Dupuytren's disease did not escape the beady eyes of the 360 Editorial Board this month. Not content with limiting trials of splinting to post-operative management, researchers in Kingston (Canada) designed and executed a study to establish what role, if any, splintage has in non-operative management of Dupytren's disease. Many surgeons routinely splint post-operatively without regard to whether there may actually be a deleterious effect, hoping that a device will help prevent an early return of a flexion contracture. The balance of risk and benefit may well be different when there is no surgical insult and the aim is simply to prevent progression. The research team reasoned that surgical treatment of proximal interphalangeal joint contractures (PIPJ) is complex, results are not guaranteed and are associated with prolonged rehabilitation. They therefore designed an alternate regimen of night splinting and stretching to prevent progression of early contractures. They report a small prospective cohort series (Level III evidence) examining the efficacy of this new treatment strategy. Of the 13 patients enrolled, 12 were able to tolerate the treatment. Patients had a mean age of 69 and were followed up for a year. Outcomes were quantified as range of movement measurements for each joint. The 12 patients reported in the study significantly improved their extension $\operatorname{arc}$ by $15^{\circ}$ by final follow-up. ${ }^{1}$ Here at 360 , we feel it stands to reason that the use of a splint would hold the contracture, and the study on the face of it makes sense (face validity). We can see how patients who wish to maintain their current level of function and not submit to surgery, with the associated risks this entails, would benefit greatly from this treatment. In light of this study (and one reported in last month's 360) there is obviously a bit of a dichotomy here; splints are helpful without surgery, but may not be helpful with surgery. We hope patients will not be so taken with their splints that they still want to use them when they progress to surgery!

\section{Salvage of the unsalvageable}

- There is a plethora of well conducted randomised controlled trials establishing the best primary intervention for various groups of patients with carpometacarpal joint osteoarthritis. While some surgeons have differing opinions, the majority would agree that trapeziectomy without interposition or suspension has been shown in most cases to be equal to other options. What then to do with the failures? A research team from London (UK) have reported a case series of salvaged, failed, trapeziectomies through the use of silastic implants. They present a retrospective series of ten cases (Level IV evidence). The paper's recommendation of the insertion of a silastic spacer for a patient presenting with a failed trapeziectomy, provides a reasonable solution in a series of ten patients reviewed at 53 months. This revision surgery was performed in patients who still had ongoing symptoms at around three years following primary surgery. The authors report that nine patients clinically improved as measured by self-reported pain, pinch grip, ADLS, and satisfaction. ${ }^{2}$ The results of this small series are encouraging, although we are slightly perplexed as trapeziectomy remains the preferred salvage option for failed arthroplasty, and has generally better functional results than other options. We would venture (with tongue placed firmly in cheek) to summarise as follows: the solution for a failed trapeziectomy is to use an implant, and the solution for a failed implant is a trapeziectomy!

\section{A close shave for malunions}

- In a paper that is so controversial that the concept caused heated tempers and raised blood pressure around the 360 office we were certainly interested to see a novel approach to treating malunion. The interplay between stability and joint congruency has not had much attention in the medical press recently, but 'how good a reduction is good enough?', has certainly had its fair share of the press. Recognised teaching includes general rules of thumb as diverse as: $50 \%$ of the articular cartilage width, $2 \mathrm{~mm}, 1 \mathrm{~mm}$, and perfect appearance on x-ray, to trauma surgeons who just aren't satisfied until they can no longer feel the fracture. We have never before, however, seen or heard of any units proposing burring away the incongruity in the case of intra-articular malunion. We were unashamedly as gobsmacked here at $360 \mathrm{HQ}$ as it is possible to be. Reasoning that the radiocarpal joint has an unusual ability to maintain function even in the presence of radiological osteoarthritis and faced with a small symptomatic group of patients, the research group from Santander (Spain) ran a small prospective study (Level III evidence) evaluating the practice of arthroscopic debridement of irregular articular surface, sacrificing up to $60 \%$ of the chondral surface for a smooth joint. The surgical team treated ten patients between the ages of 17 and 68 with a symptomatic malunion and evidence of early carpal erosion. The surgeon removed on average a third of the joint surface (20\% to $60 \%$ ). They report immediate relief of symptoms. At over two years' follow-up extension improved from a mean of $24^{\circ}$ to $54^{\circ}$ ), grip strength from $47 \%$ to $89 \%$ and DASH score from 74 to 18. ${ }^{3}$ These really are extraordinary results in all parameters from movement to patient outcome measures. The research team defend this aggressive and controversial stance, suggesting that the only other surgical option would be a salvage procedure. We wonder, however, (given that grip strength which is alignment- and not congruityrelated also improved dramatically) if much of this improvement could have been obtained with time and rehabilitation without removing 
much of the patient's articular cartilage. Call us old-fashioned but here at 360 we much prefer our patients with their cartilage and we won't become trigger-happy on the arthroscope just yet, especially not in 17-year-olds.

\section{A more classic approach to malunion}

- Although not always known for adopting a traditional approach to malunion, Jesse Jupiter and his team in Boston (USA) have come to the rescue of those who are burr-adverse when it comes to tricky-to-treat distal radial osteotomies. Using more traditional thinking the research group felt that in complex deformities in selected cases a combined intra- and extra-articular osteotomy was warranted. The research group designed a study to evaluate the efficacy of such an approach in skeletally mature patients presenting with symptomatic complex combined deformities. They recruited the patients into a prospective cohort study (Level III evidence). Eighteen patients were recruited into the study, all with a combination of extra-articular deformity $\left(\geq 15^{\circ}\right.$ volar or $\geq 10^{\circ}$ dorsal angulation or $\geq 3 \mathrm{~mm}$ shortening) and intra-articular step-off of $2 \mathrm{~mm}$ or greater. Patients were followed up to a mean of 6.5 years with a combination of clinical examination, radiological measurements and validated outcome scores (Mayo and DASH). The investigators report significant improvements in range of movement and grip strength ( $185 \%$ and $241 \%$ of pre-operative, and $89 \%$ and $84 \%$ of contralateral side, respectively). The clinical outcomes were good or excellent in $72 \%$ of cases (Mayo score) and the mean DASH score was 11. During the period of follow-up seven patients went on to develop osteoarthritis. 4 The investigators conclude that their results for combined deformity and combined osteotomy are comparable with other series of isolated intra- or extra-articular deformity and osteotomy, and conclude that the combined procedure is an acceptable treatment option.
Here at 360 we would very much agree with them.

\section{Diabetic carpal tunnel: a difficult problem}

- The diabetes epidemic, particularly in the developed West, has rekindled interest in the surgical outcomes of diabetic patients. While not exactly an appealing topic to research or publish about, it is essential that we ensure that treatments offered to diabetic patients

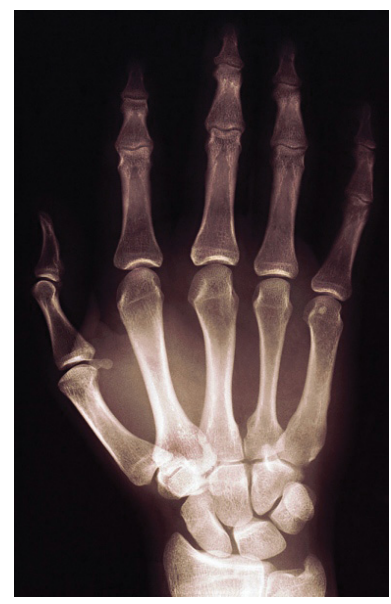

are appropriate and that we are not inadvertently placing them at excess risk of complications for often compromised benefit. Carpal tunnel decompression is one area where, intuitively, one might expect the outcomes to be poorer due to the combination of higher complication rates and the potential for concomitant diabetic neuropathy. Researchers from Szczecin (Poland) set out to establish if the risk benefit analysis was any different in diabetic patients to their peers, by investigating outcomes and complications in a diabetic and non-diabetic retrospective comparative series (Level III evidence). The research team included 386 patients, of which $11 \%$ $(n=41 / 386)$ had a diagnosis of diabetes. Patient outcome was determined with clinical evaluation including scoring (Levine scores, monofilament testing, grip and pinch tests) at six months following surgery. The research team were unable to establish any significant differences in outcomes between the two groups in terms of any variables. ${ }^{5}$ Although one might expect the outcomes of carpal tunnel decompression to be poorer in diabetic populations, the evidence presented here is compelling because it suggests that it is not. There are enough patients included in this study for any large differences in any outcome measures to be demonstrated. Diabetic patients should be offered carpal tunnel release with the expectation of similar outcomes to those of their non-diabetic peers

\section{Capsulodesis: next to useless in DISI wrists?}

- The danger with peer review (while it doubtless maintains quality) is publication bias. None of us really like publications with negative results; we don't like writing them up, discussing them, reviewing them or publishing them. Sometimes, however, knowing what not to do is hugely important. Researchers from Munich (Germany) have investigated the value of dorsal capsular plication and capsulodesis in static scapholunate instability. They designed a retrospective cohort study (Level IV evidence) to evaluate their experience of soft-tissue capsulodesis as a treatment for established scapholunate instability. The authors included 59 patients who underwent the surgery. Patients were reviewed at a mean of eight years (4 to 12) following surgery, and outcomes were assessed through radiographic evaluation, clinical examination and outcome scores (Mayo Wrist Score and DASH score). During the period of the study $14 \%$ of patients required revision surgery. The DASH and Mayo wrist scores improved significantly immediately following surgery but were only 28 and 61 respectively at final follow-up. On final radiographs $40 \%$ of patients had evidence of osteoarthritic changes and the mean carpal height index had fallen significantly from 1.53 to $1.48 .^{6}$ The authors concluded that despite short-term benefits, capsulodesis did not maintain carpal mechanics over time. Here at 360 we take our proverbial hat off to the authors, reviewers and editor of the journal for overcoming publication bias and printing this important paper. With long-term follow-up it is clear that capsulodesis does not cut the mustard with carpal instability, failing to maintain the correction of the intercalated segment. Short-term follow-up, of course, would have painted a very different picture!

\section{A wrist from a fibula?} Whatever next...

- Microsurgical techniques open up a huge range of potential novel surgical operations. One of the most common forms of vascularised bone graft is vascularised fibula, a workhorse which can be used to address any large bone void with the expectation that the vascularised nature will result in rapid healing and the ability to utilise bulk autograft. Researchers from Kyung Hee (South Korea) used this technique for reconstruction via autograft arthroplasty for defects caused by distal radius giant cell tumours. The authors describe a novel wrist arthroplasty technique, performed with a vascularised fibular head. The surgical team performed the procedure on 12 patients with stage 2 Enneking disease. Patients were followed up for over six years and outcomes were assessed using radiological and functional scores. Although all grafts had healed by 16 weeks there were a number of complications. Skin grafting was required in five patients and joint subluxation (leading to degenerative change) was present in a further five. Functionally, the patients did rather better, achieving 57\% grip strength and a flexion arc of over $70^{\circ} .7$ The authors highlight a potentially interesting technique, however, it is not clear to us that the functional results are indeed better than wrist fusion, which is a more reliable option and does not compromise grip strength as profoundly. We wonder if a comparative series may be in order? 
The editor speaks: thumbbased osteoarthritis a further opinion

- We would draw readers' attention, not this time to an original article but to an editorial, and one we were delighted to read here at 360 , as it echoes and expands on some of our own concerns expressed on small joint replacements in the last 360. Grey Giddins draws the attention of readers of the Journal of Hand Surgery to the very poor results of multiple different implants for basal thumb osteoarthritis, and summarises the current clinical data, such as it is. The editorial highlights the issues with patients undergoing the procedure giving informed consent. Indeed, if the results are unknown, how can the patient possibly give informed consent? Until publications exist supporting an implant, consent in any reasonable way cannot be given. As they are less well established and there have been no high profile catastrophic failures such as the Capitol Hip or ASR resurfacing, regulation of hand implants has been rather neglected by the orthopaedic community and health protection agencies the world over. How the robust series of measures put into place to regulate hip and knee replacement (registry/review committee scrutiny/a set of minimal longevity requirements) could be implemented with such a paucity of data is difficult to envisage. Yet most hand implants have a far worse outcome than these so-called 'disasters', yet as the editorial comments, surgeons can still start to use a new implant with little scrutiny and, unbelievably in 2013, with no commitment to the collection of basic outcome data. ${ }^{8}$ We at 360 believe hand surgeons need to urgently take this forward in the interests of their patients.

\section{REFERENCES}

1. Larocerie-Salgado J, Davidson J. Nonoperative treatment of PIPJ flexion contractures associated with Dupuytren's disease. $J$ Hand Surg Eur Vol 2012;37:722-727.

2. Umarji SI, Arnander MW, Evans DM. The use of Swanson silastic interposition arthroplasty in revision thumb-base surgery for failed trapeziectomy; a case series of 10 patients. J Hand Surg Eur Vol 2012;37:632-636.

3. Del Piñal F, Klausmeyer $M$, Thams C, Moraleda E, Galindo C. Arthroscopic resection arthroplasty for malunited intra-articular distal radius fractures. J Hand Surg Am 2012;37:2447-2455.
4. Buijze GA, Prommersberger KJ, González Del Pino J, Fernandez DL, Jupiter JB. Corrective osteotomy for combined intra- and extraarticular distal radius malunion. Hand Surg Eur Vol 2012; (Epub ahead of print) PMID: 22939826.

5. Zyluk A, Puchalski P. A comparison of outcomes of carpal tunnel release in diabetic and non-diabetic patients. I Hand Surg Eur Vol 2012;(Epub ahead of print) PMID: 23221178.

6. Megerle K, Bertel D, Germann G, Lehnhardt $M$, Hellmich $S$. Long-term results of dorsal intercarpal ligament capsulodesis for the treatment of chronic scapholunate instability. $J$ Bone Joint Surg [Br] 2012;94-B:1660-1665.

7. Chung DW, Han CS, Lee JH, Lee SG. Outcomes of wrist arthroplasty using a free vascularized fibular head graft for Enneking stage II giant cell tumors of the distal radius. Microsurgery 2012; (Epub ahead of print) PMID: 22976356.

8. Giddins G. Thumb arthroplasties. J Hand Surg Eur Vol 2012;37:603-604 